Classification

Physics Abstracts

$32.80-34.00-42.50$

\title{
Dressed atom approach to collisional redistribution
}

\author{
S. Reynaud and C. Cohen-Tannoudji \\ Laboratoire de Spectroscopie Hertzienne de l'Ecole Normale Supérieure et Collège de France, \\ 24, rue Lhomond, F 75231 Paris, France
}

( Reçu le 8 février 1982, accepté le 22 mars 1982)

\begin{abstract}
Résumé. - La description de la fluorescence de résonance par la méthode de l'atome habillé est généralisée pour inclure les effets collisionnels. Ces effets s'interprètent comme une modification par les collisions de la cascade radiative de l'atome habillé. Toutes les caractéristiques des spectres d'absorption et d'émission sont exprimées en fonction des paramètres de relaxation, de type $T_{1}$ et $T_{2}$, de l'atome habillé. On analyse le domaine de validité de cette approche et on montre qu'il est plus large que celui des équations de Bloch optiques. Les paramètres de relaxation sont reliés à la matrice $S$ de collision. En utilisant une représentation de spin, on les met également sous la forme de transformées de Fourier d'une fonction de corrélation de la perturbation collisionnelle.
\end{abstract}

\begin{abstract}
The dressed atom approach to resonance fluorescence is extended to include collisional effects. These effects appear as a collision induced modification of the radiative cascade of the dressed atom. All the characteristics of absorption and emission spectra are expressed in terms of the $T_{1}$ and $T_{2}$ type relaxation parameters of the dressed atom. The domain of validity of this approach is investigated and is shown to be larger than that of the optical Bloch equations. The relaxation parameters are related to the collision $S$ matrix. By using a spin representation, they are also expressed in terms of the Fourier transform of some correlation function of the collisional perturbation.
\end{abstract}

1. Introduction - The effect of collisions on absorption and emission spectra of atoms has always attracted the attention of physicists [1-32].

In absorption studies, several interesting questions may be asked about the absorption profile which is recorded when the frequency $\omega_{\mathrm{L}}$ of the incident light beam is scanned through the atomic frequency $\omega_{0}$ of the absorbing vapour : evaluation of the pressure broadening and of the pressure shift of the line; understanding of the lineshape in the "far wings" $\left(\left|\omega_{\mathrm{L}}-\omega_{0}\right| \gtrsim \tau_{\mathrm{c}}^{-1}\right.$ where $\tau_{\mathrm{c}}$ is the collision time), i.e. outside the " impact domain " $\left(\left|\omega_{\mathrm{L}}-\omega_{0}\right| \lesssim \tau_{\mathrm{c}}^{-1}\right)$ where this lineshape is lorentzian; effect of an increase of the light intensity...

More recently, a great deal of theoretical and experimental activity has been devoted to the study of collisional redistribution [10-13, 17-20, 22, 24-32]. In this case, the frequency $\omega_{\mathrm{L}}$ of the incident light beam is fixed, and one studies how this frequency is redistributed in the spectrum of the scattered light. New collision induced lines appear in the emission spectrum. For example, for a two level atom, a fluorescence component at $\omega_{0}$ appears in addition to the well known Rayleigh component at $\omega_{\mathrm{L}}$ (Fig. 1).
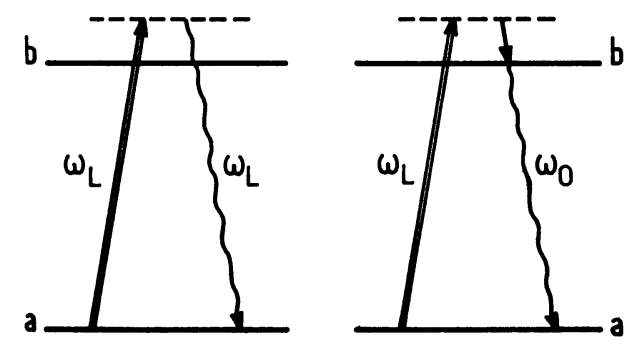

Fig. 1. - Perturbative interpretation of Rayleigh scattering (left diagram) and collisional redistribution (right diagram).

This fluorescence component can be interpreted as due to a transfer of atoms from the lower state a to the upper state $b$ through the combined effect of the exciting light (double arrow) and collisions (simple arrow).

Saturation effects associated with an increase of the laser intensity have been also investigated. It is well known that, in absence of collisions, the fluorescence spectrum is a triplet for a two level atom [33-35 and references listed in 36]. The positions, widths and 
weights of the three components of this triplet are sensitive to collisions [12]. One method for studying saturation effects is based on a non perturbative solution of the so called « optical Bloch equations" :

$$
\begin{aligned}
& \frac{\mathrm{d}}{\mathrm{d} t} \sigma_{\mathrm{a}}=-i\left[\left(H_{\mathrm{a}}-D E_{\mathrm{L}}\right.\right.\left.\left.\cos \omega_{\mathrm{L}} t\right), \sigma_{\mathrm{a}}\right]- \\
&-\left(\mathcal{F}_{\mathrm{rad}}+\mathcal{F}_{\mathrm{coll}}\right) \sigma_{\mathrm{a}}
\end{aligned}
$$

giving the rate of variation of the atomic density matrix $\sigma_{\mathrm{a}}$ as a sum of independent rates associated with the free atomic evolution (atomic hamiltonian $\left.H_{\mathrm{a}}\right)$, the interaction of the dipole moment $D$ with the laser electric field $E_{\mathrm{L}} \cos \omega_{\mathrm{L}} t$ (first line of 1.1), the radiative damping due to spontaneous emission and the collisional relaxation (second line). The relaxation matrices $\mathscr{F}_{\text {rad }}$ and $\mathcal{F}_{\text {coll }}$ are taken to be the same as in the absence of laser irradiation [17-19]. The domain of validity of equation (1.1) is given by :

$$
\omega_{1} \tau_{\mathrm{c}} \ll 1 \text { and }|\delta| \tau_{\mathrm{c}} \ll 1
$$

where $\omega_{1}=d E_{\mathrm{L}}$ is the Rabi frequency (product of the atomic dipole moment $d$ by the laser electric field $E_{\mathrm{L}}$ ), $\delta=\omega_{\mathrm{L}}-\omega_{0}$ is the detuning between the laser and atomic frequencies $\omega_{\mathrm{L}}$ and $\omega_{0}, \tau_{\mathrm{c}}$ the collision time $\left({ }^{1}\right)$.

Outside the domain defined by conditions (1.2), it is no longer possible to add independent rates of variation for the effect of collisions and the effect of the laser excitation : the laser atom coupling cannot be ignored during the collision time. Any derivation of the absorption and redistribution spectra must therefore include a correct description of the collision dynamics in the presence of the laser irradiation. Among the various theoretical methods which have been used ( $S$ matrix, tetradic scattering formalism, projection operators, ...), a particularly fruitful point of view was developed by Lisitsa and Yakovlenko [15] who considered that collisions induce transitions between the energy levels of the compound system " atom + laser photons interacting together". This approach initially developed for absorption spectra [15, 23], has been also applied to collisional redistribution, at least for certain characteristics of the spectra and for certain regimes [18, 26, 27, 28].

In this paper, we would like to analyse in more details the physical content of such a "dressed atom " approach and its domain of validity $\left({ }^{2}\right)$. For the sake of simplicity and in order to emphasize the basic ideas, we restrict the discussion to a simple model of collisions involving two level atoms, neglecting quenching, polarization redistribution, velocity changing collisions and treating classically the atomic

(1) Conditions (1.2) define what is often called the "impact region». We will come back to this terminology (see $\S 4.3$ ).

$\left({ }^{2}\right)$ A preliminary report of this work has been presented at the fifth laser spectroscopy conference [42]. motion. Within such a simple model, we show that all characteristics of absorption and emission spectra (and not only some of them) can be calculated and interpreted in terms of simple physical pictures. We point out also that such a treatment is not limited to certain regimes, as it is the case for the optical Bloch equations. A further motivation of this paper is to investigate the connections between this dressed atom approach and other ones such as optical Bloch equations, collision $S$ matrix, spin relaxation in the rotating frame, ... which provides useful physical insights in the problem.

The formulation of collisional redistribution presented in this paper is actually a straightforward extension of a previous treatment of collisionless resonance fluorescence in which fluorescence photons are considered as photons spontaneously emitted by the "dressed atom " (compound system atom plus laser photons) cascading downwards its energy diagram [35-36]. The emission frequencies are simply the Bohr frequencies of the dressed atom. The widths of the lines are the damping rates of the corresponding components of the dipole moment. The weights appear as products of transition rates by the populations of the emitting levels, these populations being given by simple rate equations. We show in this paper (section 3) that such a simple physical interpretation of the emission spectrum remains valid when the effect of collisions is incorporated as a supplementary relaxation mechanism of the dressed atom, entirely described by three parameters in terms of which all the characteristics of the spectra can be expressed. The first of these three parameters, $w$, which is identical to the " optical collision rate " introduced by Lisitsa and Yakovlenko [15] describes a population redistribution between the dressed states. Populations are given by rate equations including collisional as well as radiative processes. One gets in this way a simple understanding of the effect of collisions on the weights of the lines. The two last parameters $\kappa$ and $\xi$ describe the collisional damping and frequency shift of some off-diagonal matrix elements of the dressed atom density matrix.

Other types of dressed states are sometimes considered in the literature $[18,19]$. A unitary transformation corresponding to the diagonalization of the interaction of the two level atom with a $c$-number laser field leads to the introduction of two dressed states. Such an approach, with only two dressed states instead of the ladder of energy levels considered above and resulting from a quantum description of the laser field, does not provide the picture of the radiative cascade and the simple interpretation of the weights as products of populations by transition rates. However, if a spin representation of the two level atom is used, these two dressed states appear as the up and down states of the fictitious spin along the direction of the effective field $\mathbf{H}_{\text {eff }}$ in the rotating frame and the collisional parameters $w, \kappa$ and $\xi$ intro- 
duced above can be interpreted as the $T_{1}$ and $T_{2}$ type relaxation parameters of the fictitious spin precessing around $\mathbf{H}_{\text {eff }}$ (section 2). In this paper, we will use as often as possible this geometrical representation of the problem. This will allow us for example to understand why collisions, which are supposed elastic for the bare atom, become inelastic for the dressed one, or to interpret with geometrical arguments the separation between the various regimes. Furthermore, the spin representation gives the possibility of formulating the problem of collisional relaxation in terms of spin relaxation induced by a fluctuating magnetic field. Such a formulation will suggest some new insights concerning the structure of the expressions giving $w$ and $\kappa$ (section 5).

We also show in this paper how the dressed atom approach presented here is related to the collision $S$ matrix (section 4). The dressed atom relaxation equations, given in section 3 , can be derived from the $S$ matrices corresponding to elementary collisions by a coarse grained time average valid in the binary collision regime considered in this paper. We make explicit the various approximations which are used in this derivation and discuss the corresponding restrictions on the results presented in section 3 and deduced from the relaxation equations. When the elementary collisions are independent of the laser irradiation (condition 1.2), the $S$ matrices of the bare atom and of the dressed one are simply related, which leads to simple expressions giving $w, \kappa$ and $\xi$ in terms of the collisional damping rate $\gamma$ and frequency shift $\eta$ of the bare atom dipole moment.

2. The spin representation. - In order to use geometrical arguments throughout the paper, we first introduce in this section the spin representation of the problem.

2.1 Fictitious MAGNETIC FIELDS CORRESPONDING TO THE VARIOUS COUPLINGS. - It is well known that any two level system can be represented by a fictitious spin 1/2. The up and down states of such a spin (with respect to a given direction $\mathrm{O} z$ ) correspond respectively to the upper and lower atomic states $\mathrm{b}$ and $\mathrm{a}$. The atomic hamiltonian, responsible for the splitting $\omega_{0}$ between $a$ and $b$, is described by the effect of a magnetic field $\mathbf{H}_{0}$ parallel to $\mathrm{O} z$ and with magnitude $\omega_{0}$ (for the sake of simplicity, the fields will be expressed in units of Larmor frequency).

We consider in this paper elastic collisions which cannot induce transitions between the two atomic states $\mathrm{a}$ and $\mathrm{b}$ (the quenching is supposed negligible). In other words, collisions just produce energy shifts of the two states a and $b$, and, consequently, a time dependent variation $\Delta \omega_{0}(t)$ of the atomic splitting $\omega_{0}$ (responsible for a phase shift of the atomic dipole moment occurring after each elementary collision). If follows that, in the spin representation, collisions can be described by the effect of an additional time dependent magnetic field $\mathbf{h}(t)$, parallel to $\mathbf{H}_{0}$, and changing the length $\omega_{0}$ of $\mathbf{H}_{0}$ by an amount $\Delta \omega_{0}(t)$. The parallelism between $\mathbf{H}_{0}$ and $\mathbf{h}(t)$ geometrically reflects the elastic character of collisions : the collisional field $\mathbf{h}(t)$ cannot flip the spin along the direction of $\mathbf{H}_{0}$ (only $T_{2}$ type relaxation).

The laser atom coupling is described in the spin representation by the effect of a circularly polarized field $\mathbf{H}_{1}$, perpendicular to $\mathbf{H}_{0}$, rotating around $\mathbf{H}_{0}$ at the laser frequency $\omega_{\mathbf{L}}$ (we make the usual « rotating-wave approximation "), and having a magnitude $\omega_{1}$ (Rabi frequency). The usual transformation to the " rotating frame » (frame $\mathrm{OXYZ}$ rotating around $\mathrm{H}_{0}$ at the frequency $\omega_{\mathrm{L}}$ ) makes $\mathbf{H}_{1}$ time independent (for example parallel to $\mathrm{O} X$ ), reduces $\mathbf{H}_{0}=\omega_{0} \hat{Z}$ to $\mathbf{H}_{0}^{\prime}=\left(\omega_{0}-\omega_{\mathrm{L}}\right) \hat{Z}$ and leaves $\mathbf{h}(t)$ unchanged (Fig. 2a).

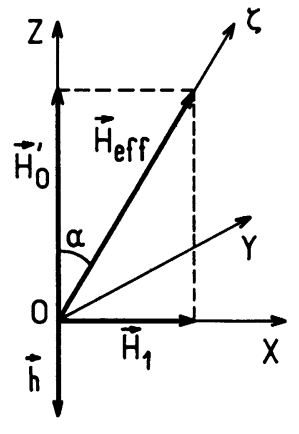

(a)

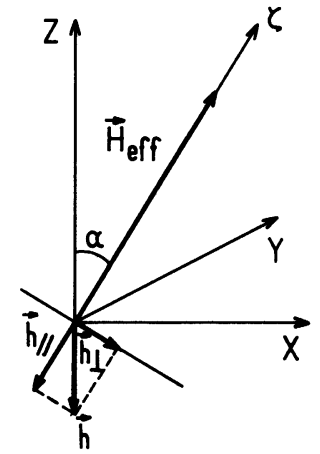

(b)
Fig. 2. $-a)$ Various magnetic fields acting upon the spin in the rotating frame $O X Y Z: \mathbf{H}_{0}^{\prime}$ (magnitude $\omega_{0}-\omega_{\mathrm{L}}$ ) is associated with the free evolution, $\mathbf{H}_{1}$ (magnitude $\omega_{1}$ ) with the laser-atom coupling, and $h$ (magnitude $\Delta \omega_{0}(t)$ ) with the collisional perturbation. $b$ ) The spin precesses around the effective field $\mathbf{H}_{\text {eff }}=\mathbf{H}_{0}^{\prime}+\mathbf{H}_{1}$. The collisional field $\mathbf{h}$ has a component $\mathbf{h}_{\perp}$, perpendicular to $\mathbf{H}_{\text {eff }}$ which can flip the $\operatorname{spin}\left(T_{1}\right.$ type relaxation).

In absence of collisions $(\mathbf{h}(t)=0)$, the spin precesses around the total static field, the so called " effective field » :

$$
\mathbf{H}_{\text {eff }}=\mathbf{H}_{0}^{\prime}+\mathbf{H}_{1}
$$

with a Larmor frequency :

$$
\Omega=\left|\mathbf{H}_{\text {eff }}\right|=\left(\omega_{1}^{2}+\delta^{2}\right)^{1 / 2} .
$$

The two spin energy levels in the rotating frame are therefore separated by $\Omega$ and they correspond respectively to the up and down states $|+\zeta\rangle$ and $|-\zeta\rangle$ along the $\mathbf{O} \zeta$ direction of $\mathbf{H}_{\text {eff }}$ (Fig. $2 a$ ) which makes with $\mathrm{OZ}$ an angle $\alpha$ such that

$$
\operatorname{cotg} \alpha=\left(\omega_{0}-\omega_{\mathrm{L}}\right) / \omega_{1}=-\delta / \omega_{1}
$$

and

$$
0 \leqslant \alpha<\pi \text {. }
$$


Actually, the two spin states $| \pm \zeta\rangle$ are the spin representatives of the two dressed states considered in several papers.

\subsection{GeOMETRICAL CONSIDERATIONS ABOUt THE COL-} LISIONAL RELAXATION. - In order to study the collisional relaxation in presence of laser irradiation, one must evaluate the effect of the collisional field $\mathbf{h}(t)$ on the motion of the spin precessing around $\mathbf{H}_{\text {eff }}$. This suggests to decompose $\mathbf{h}(t)$ in two components $\mathbf{h}_{\|}$and $\mathbf{h}_{\perp}$ respectively parallel and perpendicular to $\mathbf{H}_{\text {eff }}$ (Fig. 2b). With such a geometrical representation, some important points clearly appear. The component $\mathbf{h}_{\perp}$ can now flip the spin along the direction $\mathrm{O} \zeta$ (transitions can be induced between the two energy levels $| \pm \zeta\rangle$ ). The collisional relaxation which was initially of a pure $T_{2}$ type acquires a $T_{1}$ type character when the laser irradiation is introduced. A very simple physical meaning can also be given to the parameter $\Omega \tau_{\mathrm{c}}$ in terms of which the domain of validity of optical Bloch's equation can be defined (conditions 1.2 are equivalent to $\Omega \tau_{\mathrm{c}} \ll 1$ ). $\Omega \tau_{\mathrm{c}}$ is nothing but the angle of precession of the spin around $\mathbf{H}_{\text {eff }}$ during the collision time. Condition $\Omega \tau_{\mathrm{c}} \ll 1$ means that the precession of the spin is negligible during the time $\tau_{\mathrm{c}}$. In other words, the laser atom coupling can be ignored during the collision and this explains why the same relaxation matrix $\mathcal{F}_{\text {coll }}$ as in the absence of the laser can be still used (provided of course that it is transformed by the purely geometrical rotation bringing $\mathrm{O} Z$ along $\mathrm{O} \zeta$, when the basis $\{| \pm \zeta\rangle\}$ in used). On the contrary, when $\Omega \tau_{\mathrm{c}} \gtrsim 1$, the collision dynamics is affected by the precession around $\mathbf{H}_{\text {eff }}$. We will come back on this problem in section 5 .

2.3 FLUORESCENCE SPECTRUM. - We now come back to our initial problem which is to understand the fluorescence spectrum in the presence of collisions. In order to find the positions of the lines, we can first neglect the radiative and collisional damping and look at the frequencies appearing in the evolution of the various components of the spin. In the rotating frame, the $\zeta$ component of the spin is static, whereas the two other components, perpendicular to $O \zeta$, oscillate at $\pm \Omega$. Coming back from the rotating frame to the laboratory frame transforms $0, \pm \Omega$ into $\omega_{\mathrm{L}}, \omega_{\mathrm{L}} \pm \Omega$ which are just the well known three frequencies of the fluorescence triplet [37]. If these three lines remain well resolved in the presence of damping, this means that their splitting $\Omega$ is large compared to the collisional and radiative widths. In other words, the spin precesses several times around $\mathbf{H}_{\text {eff }}$ during the relaxation time. This implies that the relaxations of the components of the spin respectively parallel and perpendicular to $\mathbf{H}_{\text {eff }}$ are decoupled and correctly described by $T_{1}$ and $T_{2}$ relaxation times. Since the central inelastic component of the fluorescence spectrum is related to the motion of the component of the spin parallel to $\mathbf{H}_{\text {eff }}$ in the rotating frame, it follows that this line has a width given by $1 / T_{1}$. On the other hand, the two sidebands at $\omega_{\mathrm{L}} \pm \Omega$, which are generated by the components of the spin precessing around $\mathbf{H}_{\text {eff }}$, have a width $1 / T_{2}$. It remains to understand the weights of the lines. It seems then difficult to extract from the spin representation some simple interpretations for the structure of the mathematical results which are obtained. This is mainly due to the fact that the spin representation, which is very well suited for analysing the motion and the damping of the atomic system, does not describe how photons are absorbed and emitted. We show in the next section how the picture of the radiative cascade of the dressed atom provides a simple solution to this problem while keeping the main advantages of the previous discussion.

3. The dressed atom approach. - In this section we present the dressed atom approach to the problem of collisional redistribution. We first recall the interpretation of collisionless resonance fluorescence in terms of a radiative cascade of the dressed atom $(\S 3.1)$ (a more extensive discussion of this problem can be found in [35-36]). We then describe the effects of collisions as producing an additional relaxation mechanism of the dressed atom $(\S 3.2)$. We finally show that the various characteristics of the fluorescence and absorption spectra can be expressed in terms of the relaxation parameters ( $\S 3.3$ to 3.5$)$.

\subsection{Collisionless Resonance FluOResCence as A} RADIATIVE CASCADE OF THE DRESSED ATOM. - When the laser field is considered as a quantum system, the energy levels of the compounds system « atom plus laser photons interacting together " (the " dressed atom ") are labelled by two quantum numbers : a and $\mathrm{b}$ for the lower and upper atomic states, $n$ for the number of laser photons. They form two dimensional manifolds separated by $\omega_{\mathrm{L}}$ (Fig. $3 a$ ). The two states $|\mathrm{a}, n+1\rangle$ and $|\mathrm{b}, n\rangle$ of the manifold $\varepsilon_{n}$ are separat-

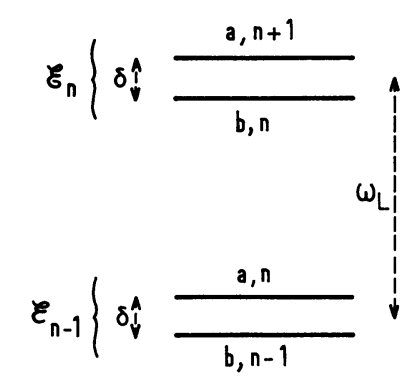

(a)

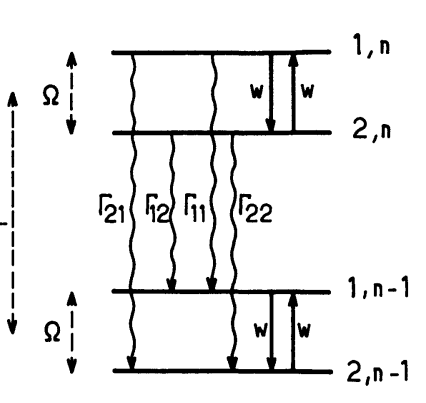

(b)
Fig. 3. - a) Unperturbed manifolds of the combined system atom plus laser photons. $b$ ) Dressed states. Radiative and collisional transitions between the dressed states are respectively represented by wavy and straight arrows. 
ed by the detuning $\delta=\omega_{\mathrm{L}}-\omega_{0}$ and coupled (coupling $\omega_{1} / 2$ ) by the laser atom interaction (the atom can jump from a to b by absorbing a laser photon) $\left({ }^{3}\right)$. The dressed states $|1, n\rangle$ and $|2, n\rangle$ which diagonalize the total hamiltonian are represented in figure $3 b$. Their splitting is :

$$
\Omega=\left[\omega_{1}^{2}+\delta^{2}\right]^{1 / 2}
$$

and the wave functions are given by :

$$
\begin{aligned}
& |1, n\rangle=\cos \theta|\mathrm{b}, n\rangle+\sin \theta|\mathrm{a}, n+1\rangle \\
& |2, n\rangle=-\sin \theta|\mathrm{b}, n\rangle+\cos \theta|\mathrm{a}, n+1\rangle
\end{aligned}
$$

with

$$
\begin{gathered}
\operatorname{cotg} 2 \theta=-\delta / \omega_{1} \\
0 \leqslant 2 \theta<\pi .
\end{gathered}
$$

It is interesting to relate the dressed atom states to the fictitious spin states of the previous section. The up and down spin states along $\mathrm{OZ}$ (in the rotating frame) are the spin representatives of the uncoupled states $|\mathrm{b}, n\rangle$ and $|\mathrm{a}, n+1\rangle$ whereas the up and down states along the direction $O \zeta$ of the effective field correspond to the dressed states $|1, n\rangle$ and $|2, n\rangle$. They have the same energy splitting $\Omega$ and their state expansions are identical since $2 \theta$ is equal to the angle $\alpha$ of the rotation from $\mathrm{OZ}$ to $\mathrm{O} \zeta$ (see eq. (2.3)). The dressed atom energy diagram is therefore an infinite ladder of equidistant manifolds, each of these manifolds being identical to the energy diagram of the fictitious spin.

The coupling of the atom with the empty modes of the electromagnetic field is responsible for the emission of fluorescence photons which can be considered as photons spontaneously emitted by the dressed atom. Emission frequencies therefore correspond to the allowed transitions between dressed states. The only non zero matrix element of the atomic dipole $D$ between the uncoupled states is :

$$
d=\langle\mathrm{a}, n|D| \mathrm{b}, n\rangle \text {. }
$$

From the expression (3.2) of the dressed states, one deduces that $D$ couples dressed states belonging to adjacent manifolds $\varepsilon_{n}$ and $\varepsilon_{n-1}$

$$
\langle\mathrm{i}, n-1|D| \mathrm{j}, n\rangle=d_{\mathrm{ij}}
$$

with

$$
\begin{gathered}
d_{11}=d_{22}=d \cos \theta \sin \theta \\
d_{12}=-d \sin ^{2} \theta, \quad d_{21}=d \cos ^{2} \theta .
\end{gathered}
$$

( $\left.{ }^{3}\right)$ Strictly speaking, the coupling between $|\mathrm{a}, n+1\rangle$ and $|\mathrm{b}, n\rangle$ is proportional to $\sqrt{n+1}$. But we use the dressed atom energy diagram in a domain of values of $n$ having a width $\Delta n$ much smaller than the average value $\bar{n}$ (quasi classical state of the laser field), so that the variation with $n$ of all physical quantities will be neglected in this interval.
As a consequence, spontaneous transitions (wavy arrows on figure 3 ) occur at the three frequencies $\omega_{\mathrm{L}}+\Omega$ (transition from $|1, n\rangle$ to $|2, n-1\rangle$ with a rate $\left.\Gamma_{21}\right), \omega_{\mathrm{L}}-\Omega\left(|2, n\rangle \rightarrow|1, n-1\rangle\right.$; rate $\left.\Gamma_{12}\right)$ and $\omega_{\mathrm{L}}(|1, n\rangle \rightarrow|1, n-1\rangle$ and $|2, n\rangle \rightarrow|2, n-1\rangle$; rates $\Gamma_{11}$ and $\Gamma_{22}$ ). This explains the triplet structure of the fluorescence spectrum and the positions of the lines. The values of the rates are deduced from the dipole matrix elements $(3.6)\left({ }^{4}\right)$ :

$$
\begin{gathered}
\Gamma_{\mathrm{ij}} \sim\left|d_{\mathrm{ij}}\right|^{2} \\
\Gamma_{11}=\Gamma_{22}=\Gamma \cos ^{2} \theta \sin ^{2} \theta=\Gamma \omega_{1}^{2} / 4 \Omega^{2} \\
\Gamma_{12}=\Gamma \sin ^{4} \theta=\Gamma(\Omega+\delta)^{2} / 4 \Omega^{2} \\
\Gamma_{21}=\Gamma \cos ^{4} \theta=\Gamma(\Omega-\delta)^{2} / 4 \Omega^{2}
\end{gathered}
$$

( $\theta$ is related to $\omega_{1}$ and $\delta$ by 3.3).

\subsection{COLlisional RELAXATION OF THE DRESSED ATOM.} - The effect of collisions is to produce a collisional relaxation of the dressed atom which is described (in the case of well resolved lines) by relaxation parameters corresponding respectively to a redistribution of the dressed atom populations ( $T_{1}$ type relaxation) and to a damping and a frequency shift of dressed atom coherences ( $T_{2}$ type relaxation).

The population redistribution may be described by the equations :

$$
\begin{gathered}
\frac{\mathrm{d}}{\mathrm{d} t} \sigma_{1 n, 1 n}=w\left(\sigma_{2 n, 2 n}-\sigma_{1 n, 1 n}\right) \\
\frac{\mathrm{d}}{\mathrm{d} t} \sigma_{2 n, 2 n}=-\frac{\mathrm{d}}{\mathrm{d} t} \sigma_{1 n, 1 n}
\end{gathered}
$$

( $\sigma$ is the dressed atom density matrix and

$$
\left.\sigma_{\mathrm{in}, \mathrm{j} n^{\prime}}=\left\langle\mathrm{i}, n|\sigma| \mathrm{j}, n^{\prime}\right\rangle\right) .
$$

Collisions tend to equalize the populations within each manifold. The rates $w$ from $|1, n\rangle$ to $|2, n\rangle$ and from $|2, n\rangle$ to $|1, n\rangle$ are equal. This is due to the fact that the energy $\hbar \Omega$ involved in a collisional transition is supposed to be much smaller than the mean kinetic energy $k T$.

A difference of nature between radiative and collisional relaxation processes clearly appears in figure 3 . Collisions are quasi elastic in the sense that they redistribute populations within each manifold (full arrows of figure 3 ) whereas spontaneous emission induces inelastic transitions at optical frequencies between adjacent manifolds. There are no collisional transitions at optical frequencies since quenching is neglected. Such a physical insight is provided by the ladder representation of the dressed atom energy

$\left({ }^{4}\right)$ Let us recall that this simple description of the radiative cascade of the dressed atom in terms of transition rates is limited to the case of well resolved lines studied in this paper. 
diagram (associated with the quantization of the laser field). The spin representation does not allow such a discussion. Furthermore, the ladder representation provides a clear interpretation of the usual picture of collisional redistribution (Fig. 1). The " virtual " level (dotted line of Fig. 1) is nothing but the dressed state $|1, n\rangle$ (which reduces to $|\mathrm{a}, n+1\rangle$ in the perturbative limit $\omega_{1} \ll \delta$ ) and the straight arrow is just the real inelastic transition from $|1, n\rangle$ to $|2, n\rangle$. Let us emphasize that the dressed atom picture of figure 3 remains valid in the non perturbative domain $\left(\omega_{1} \gtrsim \delta\right)$.

The $T_{2}$ type relaxation equations can be written by introducing two parameters $\kappa$ and $\xi$ associated with the damping and frequency shift of the coherences between $|1, n\rangle$ and $|2, n\rangle$ :

$$
\begin{aligned}
& \frac{\mathrm{d}}{\mathrm{d} t} \sigma_{1 n, 2 n}=-(\kappa+i \xi) \sigma_{1 n, 2 n} \\
& \frac{\mathrm{d}}{\mathrm{d} t} \sigma_{2 n, 1 n}=-(\kappa-i \xi) \sigma_{2 n, 1 n} .
\end{aligned}
$$

In section 4 , when deriving the equations (3.9) and (3.10) from the $S$ matrix approach, we will show that the relaxation of coherences between different manifolds is described by the same relaxation parameters $w$, $\kappa$ and $\xi$. These three parameters depend of course on $\omega_{1}$ and $\delta$.

The dressed atom picture (Fig. 3) clearly shows that the emission spectrum remains a triplet in the presence of collisions. We will now relate the weights and widths of the lines to the radiative and collisional relaxation parameters.

3.3 WeIGHTS OF THE LINES. - The weight (integrated intensity) of a given line is the product of the transition rate by the steady state population of the emitting dressed state :

$$
\begin{gathered}
J\left(\omega_{\mathrm{L}}+\Omega\right)=\Gamma_{21} \Pi_{1} \quad J\left(\omega_{\mathrm{L}}-\Omega\right)=\Gamma_{12} \Pi_{2} \\
J\left(\omega_{\mathrm{L}}\right)=\Gamma_{11} \Pi_{1}+\Gamma_{22} \Pi_{2}=\Gamma_{11}\left(\Pi_{1}+\Pi_{2}\right)
\end{gathered}
$$

where

$$
\Pi_{1}=\sum_{n} \sigma_{1 n, 1 n} \quad \Pi_{2}=\sum_{n} \sigma_{2 n, 2 n} .
$$

The steady state populations may be deduced from the normalization condition :

$$
\Pi_{1}+\Pi_{2}=1
$$

and from the detailed balance condition :

$$
\left(\Gamma_{12}+w\right) \Pi_{2}=\left(\Gamma_{21}+w\right) \Pi_{1}
$$

which expresses that the total number of transitions $|2, n\rangle \rightarrow\left|1, n^{\prime}\right\rangle$ balances the total number of transitions $|1, n\rangle \rightarrow\left|2, n^{\prime}\right\rangle$ (see Fig. 3). In absence of collisions $(w=0)$, such a condition clearly implies the equality of the weights of the two sidebands (when $\Gamma_{12} \Pi_{2}=\Gamma_{21} \Pi_{1}$, it follows from (3.11) that $\left.J\left(\omega_{\mathrm{L}}-\Omega\right)=J\left(\omega_{\mathrm{L}}+\Omega\right)\right)$. But, in general, the detailed balance condition (3.14) includes collisional as well as radiative transitions. The balance between radiative transitions is therefore supressed $\left(\Gamma_{12} \Pi_{2} \neq \Gamma_{21} \Pi_{1}\right)$ and the weights of the two sidebands are no longer equal : the fluorescence spectrum is asymmetric in the presence of collisions [17].

The dressed atom populations deduced from (3.13) and (3.14) are :

$$
\begin{aligned}
& \Pi_{1}=\left[\Gamma_{12}+w\right] /\left[\Gamma_{12}+\Gamma_{21}+2 w\right] \\
& \Pi_{2}=\left[\Gamma_{21}+w\right] /\left[\Gamma_{12}+\Gamma_{21}+2 w\right] .
\end{aligned}
$$

It may be checked by this expression that the collisions tend to equalize populations $\left(\Pi_{1}-\Pi_{2}\right.$ is smaller in the presence of than in the absence of collisions). In the particular case of a resonant excitation $(\delta=0)$, the populations are not modified by collisions since they are already equal in their absence. It follows that the spectrum remains symmetric in this case.

One gets finally the expressions of the weights in terms of the radiative rates $\Gamma_{\mathrm{ij}}$ and of the redistribution rate $w$ :

$$
\begin{aligned}
& J\left(\omega_{\mathrm{L}}+\Omega\right)=\Gamma_{21}\left[\Gamma_{12}+w\right] /\left[\Gamma_{12}+\Gamma_{21}+2 w\right] \\
& J\left(\omega_{\mathrm{L}}-\Omega\right)=\Gamma_{12}\left[\Gamma_{21}+w\right] /\left[\Gamma_{12}+\Gamma_{21}+2 w\right]
\end{aligned}
$$

$$
J\left(\omega_{\mathbf{L}}\right)=\Gamma_{11} .
$$

3. 4 LiNeSHAPES. - The linewidths are obtained as the damping rates of the corresponding components of the dipole moment [36].

The two sidebands at $\omega_{\mathrm{L}} \pm \Omega$ have a width (half width at half maximum) :

$$
L_{\mathrm{L}}=\Gamma\left(\frac{1}{2}+\cos ^{2} \theta \sin ^{2} \theta\right)+\kappa .
$$

The radiative contribution to this width is larger than the half sum $\Gamma / 2$ of the natural widths of the two involved levels because of the existence of a phase transfer in the radiative cascade downwards the dressed atom energy diagram [36]. The pressure broadening of the lateral components is just equal to $\kappa$. This is due to the fact the collisional relaxation of the optical coherences $\langle 1, n|\sigma| 2, n-1\rangle$ and $\langle 2, n|\sigma| 1, n-1\rangle$ (evolution frequencies $\omega_{\mathrm{L}}+\Omega$ and $\omega_{\mathrm{L}}-\Omega$ ) are identical to the equations (3.10) describing the relaxation of the low frequency coherences $\langle 1, n|\sigma| 2, n\rangle$ and $\langle 2, n|\sigma| 1, n\rangle$ (see the demonstration in section 4.1). For the same reason, the parameter $\xi$ gives the pressure shift of the lateral 
components (the precise positions of these components are $\left.\omega_{\mathrm{L}} \pm(\Omega+\xi)\right)$.

The total weight $J\left(\omega_{\mathrm{L}}\right)$ of the central component which is proportional to $\Pi_{1}+\Pi_{2}$ (eq. 3.11b) does not depend on collisions (eq. 3.16c). But the lineshape of this central component is very sensitive to collisions. Actually, one can show [36] that this line is a superposition of two components : an elastic component reproducing the laser spectrum without broadening and an inelastic one. Simple expressions [35] give the weights $J_{e l}$ and $J_{\text {inel }}\left(\omega_{L}\right)$ of these two components

$$
\begin{gathered}
\mathfrak{J}_{\mathrm{el}}=\Gamma_{11}\left(\Pi_{1}-\Pi_{2}\right)^{2} \\
\mathfrak{J}_{\mathrm{inel}}\left(\omega_{\mathrm{L}}\right)=4 \Gamma_{11} \Pi_{1} \Pi_{2} .
\end{gathered}
$$

Using $\Pi_{1}+\Pi_{2}=1$, one can check that the sum of these two weights gives the result $\Gamma_{11}$ of equation (3.16c).

The elastic component is reduced by collisions which tend to equalize the two populations $\Pi_{1}$ and $\Pi_{2}$. More precisely, one gets :

$J_{\mathrm{el}}=\Gamma_{11}\left(\Gamma_{12}-\Gamma_{21}\right)^{2} /\left[\Gamma_{12}+\Gamma_{21}+2 w\right]^{2}$.

The weight $J_{\text {inel }}\left(\omega_{L}\right)$ of the inelastic component of the central line is of course enhanced by collisions [since $\mathfrak{J}_{\text {inel }}\left(\omega_{\mathrm{L}}\right)+\mathfrak{J}_{\mathrm{el}}=\mathfrak{J}\left(\omega_{\mathrm{L}}\right)$ is independent of collisions].

We discuss now the width $L_{\mathrm{c}}$ of the central inelastic component by using geometrical arguments derived from the spin representation. The widths $L_{\mathrm{c}}$ and $L_{\mathrm{L}}$ are actually the damping rates of the components of the fictitious spin respectively parallel and orthogonal to the $\mathrm{O} \zeta$ direction of the effective field (one can write $\left.L_{\mathrm{c}}=1 / T_{\mathrm{I}} ; L_{\mathrm{L}}=1 / T_{2}\right)$. In order to get $L_{\mathrm{c}}$, we have therefore to determine the damping rate of :

$$
S_{\zeta}=\frac{1}{2}\left(\sigma_{1 n, 1 n}-\sigma_{2 n, 2 n}\right)
$$

which is nothing but the damping rate of the population transient regime. One gets :

$$
L_{\mathrm{c}}=\Gamma_{12}+\Gamma_{21}+2 w .
$$

Such a derivation clearly shows that the pressure broadening of the central inelastic component is directly related to the population redistribution rate $w$ since both quantities correspond to the relaxation of $S_{\zeta}$.

\subsection{COLLISIONAL ENHANCEMENT OF THE ABSORPTION} OF LASER PHOTONS. - The total number $A$ of absorbed laser photons is obviously equal to the total number of reemitted fluorescence photons so that it can be obtained simply by summing the weights of the three emission lines

$$
A=\Gamma_{11}+\Gamma_{21} \Pi_{1}+\Gamma_{12} \Pi_{2}
$$

(see eq. (3.11)). In the absence of collisions, the weights $\Gamma_{21} \Pi_{1}$ and $\Gamma_{12} \Pi_{2}$ of the two lateral components are equal so that the smaller population corresponds to the bigger transition rate (for example, $\Pi_{1}<\Pi_{2}$ corresponds to $\Gamma_{21}>\Gamma_{12}$ ). Since collisions tend to equalize populations, they enhance the smaller population which corresponds to the bigger transition rate and they reduce the bigger population which corresponds to the smaller transition rate. As a consequence, the absorption of laser photons is enhanced by collisions. In the resonant case, the populations are equal in the absence of collisions (since $\Gamma_{12}=\Gamma_{21}$ ). They remain equal in the presence of collisions and the absorption of laser photons is not modified in this case.

The general expression of $A$ in terms of the radiative rates $\Gamma_{\mathrm{ij}}$ and of the redistribution rate $w$ is deduced from (3.22) and (3.15). Writing the $\Gamma_{\mathrm{ij}}$ in terms of $\Gamma$ and $\theta$ (eq. (3.8)) leads to :

$$
A=\Gamma\left[\Gamma \cos ^{2} \theta \sin ^{2} \theta+w\right] /\left[\Gamma\left(\cos ^{4} \theta+\sin ^{4} \theta\right)+2 w\right] .
$$

4. Relations with the $S$ matrix approach. - In this section, we sketch the main lines of the derivation of the dressed atom relaxation equations presented in the previous section. Starting from the $S$ matrix associated with elementary collisions, we compute the coarse grained averaged rate of variation of the dressed atom density matrix ( $\$ 4.1$ ). Two approximations are made in this derivation : the Markov approximation and the secular approximation. We analyse (§ 4.2) the corresponding restrictions on the results deduced in section 3 from the relaxation equations. We finally make explicit the relation between the dressed atom relaxation parameters $w, \kappa, \xi$ and those of the free atom in the domain $\Omega \tau_{\mathrm{c}} \ll 1$ where optical Bloch equations are valid $(\S 4.3)$.

4.1 DeRIVATION OF THE DRESSED ATOM RELAXATION EQUATIONS FROM THE COLLISION $S$ MATRIX. - The emitting atoms are supposed to undergo successive «binary collisions". The collision time $\tau_{\mathrm{c}}$ is much shorter than the time $T_{\mathrm{c}}$ between two successive collisions of an emitting atom with perturbers

$$
\tau_{\mathrm{c}} \ll T_{\mathrm{c}} .
$$

It is therefore possible to define for each elementary collision an $S$ matrix depending on the impact parameter $b$ and the relative velocity $v$.

Let us consider a time interval $\Delta t$ such that :

$$
\tau_{\mathrm{c}} \ll \Delta t \ll T_{\mathrm{c}} .
$$

As the relaxation time $T_{\mathrm{R}}$ is certainly longer than $T_{\mathrm{c}}$, $\Delta t$ is much shorter than $T_{\mathrm{R}}\left(\Delta t \ll T_{\mathrm{R}}\right)$ which means that the overall effect of collisions is extremely weak during $\Delta t$ (this is not incompatible with the existence of « strong collisions "). As $\Delta t$ is much longer than the collision time $\tau_{\mathrm{c}}$, the variation of the density matrix during $\Delta t$ can be deduced from the $S$ matrices corres- 
ponding to the collisions occurring during $\Delta t$. More precisely if :

$$
\Delta \sigma=\sigma(t+\Delta t)-\sigma(t)
$$

( $\sigma$ density matrix in the interaction representation) one gets :

$$
\frac{\Delta \sigma}{\Delta t}=\sum_{\text {coll }}\left(S \sigma S^{+}-\sigma\right)
$$

where the summation over collisions is defined for any quantity $a(b, v)$ by :

$$
\sum_{\text {coll }} a=N_{\mathrm{p}} \int_{0}^{\infty} 2 \pi b \mathrm{~d} b \int_{0}^{\infty} v f(v) a(b, v) \mathrm{d} v
$$

$\left(f(v)\right.$ is the velocity distribution and $N_{\mathrm{p}}$ the volume density of perturbers).

The quantity $\Delta \sigma / \Delta t$ is the " coarse grained " derivative of $\sigma$, i.e. the mean value of the time derivative $\mathrm{d} \sigma / \mathrm{d} t$ over an interval $\Delta t$ much longer than the collision time $\tau_{\mathrm{c}}$ but much shorter than the relaxation time $T_{\mathrm{R}}$. The Markov approximation consists in identifying $\Delta \sigma / \Delta t$ and $\mathrm{d} \sigma / \mathrm{d} t$. One thus get the collisional master equation giving $\mathrm{d} \sigma / \mathrm{d} t$ in terms of $\sigma$ :

$$
\frac{\mathrm{d} \sigma}{\mathrm{d} t}=\sum_{\text {coll }}\left(S \sigma S^{+}-\sigma\right) .
$$

It is important to note that, when identifying $\Delta \sigma / \Delta t$ and $\mathrm{d} \sigma / \mathrm{d} t$, we neglect the non Markovian effects associated with the high frequency evolution of $\sigma$ (time scale of the order of the collision time $\tau_{\mathrm{c}}$ ). We will come back to this problem in section 4.2.

As quenching is neglected, the $S$ matrix only couples dressed states belonging to a particular manifold $\varepsilon_{n}$; the matrix elements of $S$ satisfy :

$$
\left\langle\mathrm{i}, n|S| \mathrm{j}, n^{\prime}\right\rangle=\delta_{n, n^{\prime}} S_{\mathrm{ij}}
$$

$\left(\delta_{n, n^{\prime}}\right.$ is the Kronecker symbol; the variation with $n$ of $S_{\mathrm{ij}}$ is neglected, see foot note $\mathrm{n}^{\circ} 3$ ). As a consequence, the master equation (4.6) has the following form in the dressed atom basis :

$$
\frac{\mathrm{d}}{\mathrm{d} t}\left\langle\mathrm{i}, n_{1}|\sigma| \mathrm{j}, n_{2}\right\rangle=\sum_{\mathrm{i}^{\prime} \mathrm{j}^{\prime}} R_{\mathrm{iji}^{\prime} \mathrm{j}^{\prime}}\left\langle\mathrm{i}^{\prime}, n_{1}|\sigma| \mathrm{j}^{\prime}, n_{2}\right\rangle
$$

where

$$
R_{\mathrm{iji} i^{\prime} j^{\prime}}=\sum_{\text {coll }}\left(S_{\mathrm{ii}^{\prime}} S_{\mathrm{jj}^{\prime}}^{*}-\delta_{\mathrm{ii}^{\prime}} \delta_{\mathrm{jj^{ \prime }}}\right) .
$$

The relaxation parameters $R_{\mathrm{iji} i^{\prime} \mathrm{j}^{\prime}}$ have the same magnitude as $\gamma$ and they are much smaller than $\Omega$ since the fluorescence lines are well separated :

$$
R_{\mathrm{iji}^{\prime} \mathrm{j}^{\prime}} \ll \Omega \text {. }
$$

It is therefore possible to neglect collisional couplings between density matrix elements evolving at different frequencies (secular approximation). One gets in this way the equations (3.9) describing the population redistribution and the equations (3.10) describing the damping and the frequency shift of the coherences. Since the parameters $R_{\mathrm{ij} i^{\prime} j^{\prime}}$ do not depend on $n_{1}$ and $n_{2}$, the relaxation of optical coherences $\left(n_{1}-n_{2}=1\right)$ is described by equations identical to the equations (3.9) and (3.10) (which correspond to low frequency density matrix elements $n_{1}=n_{2}$ ).

Furthermore, one obtains the expression of the relaxation parameters $w, \kappa$ and $\xi$ in terms of the collision $S$ matrix.

$$
\begin{aligned}
w & =\sum_{\text {coll }}\left|S_{12}\right|^{2} \\
\kappa+i \xi & =\sum_{\text {coll }}\left(1-S_{11} S_{22}^{*}\right) .
\end{aligned}
$$

Let us note that $w, \kappa$ and $\xi$ may be expressed in terms of two real parameters $p$ and $q$ defined by :

$$
S_{11}=\cos p \exp (-i q)
$$

From the unitarity of the $S$ matrix, (4.11) and (4.12) can indeed be written :

$$
\begin{aligned}
w & =\sum_{\text {coll }}\left(\sin ^{2} p\right) \\
\kappa & =\sum_{\text {coll }}\left(1-\cos ^{2} p \cos 2 q\right)=w+\kappa^{\prime} \\
\kappa^{\prime} & =\sum_{\text {coll }}\left(\cos ^{2} p(1-\cos 2 q)\right) \\
\xi & =\sum_{\text {coll }}\left(\cos ^{2} p \sin 2 q\right) .
\end{aligned}
$$

As it is usual in atomic line broadening theory, the coherence damping parameter $\kappa$ appears as the sum of an inelastic contribution $w$ ( $T_{1}$ type contribution to $T_{2}$ type relaxation) and of an elastic contribution $\kappa^{\prime}$ ( $T_{2}^{\prime}$ type relaxation). The inelastic contribution $w$ is just half the sum of the inelastic rates of redistribution from the two states involved $|1, n\rangle$ and $|2, n\rangle$ (since $w=(w+w) / 2)$.

\subsection{Restrictions associated With the MarkoV} aND SECUlar aPPROXIMATIONS. - We have now obtained the relaxation equations (3.9) and (3.10) describing the effect of collisions. The derivation of the fluorescence spectrum from these equations is a straightforward duplication of the calculations already done for collisionless resonance fluorescence [36]. One gets in this way the characteristics of this spectrum which have been given in section 3. These results have a limited range of validity because of the Markov and secular approximations used in the derivation of the relaxation equations $(\$ 4.1)$. We want here to evaluate this range of validity $\left({ }^{5}\right)$.

$\left({ }^{5}\right)$ In the derivation of the fluorescence spectrum from the relaxation equations, we also use the quantum regression theorem [38,34]. This is actually a further approximation but its condition of validity and its range of validity are strictly identical to those of the Markov approximation and they are therefore included in the following discussion. 


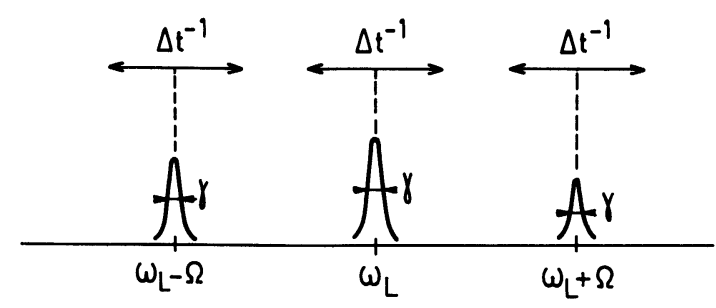

Fig. 4. - Frequency intervals over which the redistribution spectrum is correctly described in the dressed atom approach.

The Markov approximation consists in neglecting the dynamics of the density matrix $\sigma$ on time scales smaller than $\Delta t$ (i.e. of the order of $\tau_{\mathrm{c}}$ ). The redistribution spectrum derived in section 3 is therefore correct only in three intervals $(\Delta t)^{-1}$ around the three Bohr frequencies $\omega_{\mathrm{L}}+\alpha \Omega(\alpha=0, \pm 1)$ of the dressed atom (Fig. 4), i.e. for frequencies $\omega$ such that :

$$
\left|\omega-\left(\omega_{\mathrm{L}}+\alpha \Omega\right)\right| \ll\left(\tau_{\mathrm{c}}\right)^{-1}
$$

Since the widths of the lines are of the order of $\gamma$, one obtains correct results for the positions, widths and weights of the lines as soon as

$$
\gamma \ll\left(\tau_{\mathrm{c}}\right)^{-1} \text { i.e. } \tau_{\mathrm{c}} \ll T_{\mathbf{R}}
$$

which is a consequence of the condition (4.1) defining binary collisions. One gets also a correct result for the absorption of laser photons which is the integral of the fluorescence spectrum (\$3.5) since non Markovian effects have to be taken into account only outside the three intervals of figure 4 , i.e. in regions where the fluorescence is negligible.

The secular approximation consists in neglecting the non secular couplings introduced by the relaxation in the master equation (for example, couplings between populations and coherences). These non secular couplings actually give rise to corrections smaller than the secular terms by a factor of the order of $\gamma / \Omega$. However these non secular corrections may have a dispersion shape which decreases more slowly than the absorption shape of the secular terms. It follows that the non secular contributions, which are negligible near the centre of the lines, may become as important as the secular ones in the wings of the lines. More precisely, the spectrum derived in section 3 from the secular approximation is valid only in three frequency intervals

$$
\left|\omega-\left(\omega_{\mathbf{L}}+\alpha \Omega\right)\right| \ll \Omega .
$$

Since the widths of the lines are of the order of $\gamma$, one obtains correct results for the positions, widths, weights of the fluorescence lines and for the absorption as soon as

$$
\gamma \ll \Omega
$$

which is the condition defining well separated lines.
We would like to emphasize that the validity of the dressed atom approach is not restricted by the condition $\Omega \tau_{\mathrm{c}} \ll 1$ as is the case for optical Bloch equations. The advantage of the dressed atom approach is that one studies the collisions in the dressed atom interaction representation : the laser atom interaction is first diagonalized and the collisions are then included. The Markovian description of collisions is thus valid in the three intervals represented on figure 4 . On the other hand, optical Bloch equations correspond to a description of collisions in the bare atom interaction representation (same description as in the absence of laser irradiation). The results derived from optical Bloch equations can therefore be valid only if the whole redistribution spectrum is contained in an interval $(\Delta t)^{-1}$ (Fig. 5) which is just equivalent to the condition $\Omega \tau_{\mathrm{c}} \ll 1$.

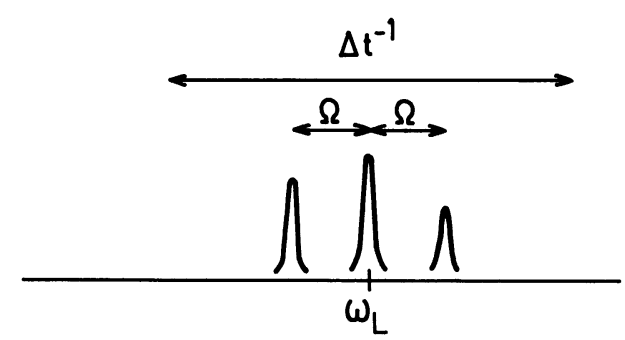

Fig. 5. - Frequency interval over which the redistribution spectrum is correctly described by optical Bloch's equations. The whole spectrum is contained in this interval only when $\Omega \tau_{\mathrm{c}} \ll 1$.

\subsection{RELATIONS WITH THE RELAXATION PARAMETERS} OF THE FREE ATOM IN THE DOMAIN $\Omega \tau_{\mathrm{c}} \ll 1$. - The purpose of this section is to compare the dressed atom relaxation equations and the optical Bloch equations in the domain $\Omega \tau_{\mathrm{c}} \ll 1$ where they are both valid. It must then be possible to relate the parameters $w, \kappa, \xi$ describing the collisional relaxation of the dressed atom to the usual parameters associated with the bare atom. We will find such a relation by using a method based on the $S$ matrix approach. We will also give a geometrical interpretation of the results.

We first make explicit the relaxation parameters of the bare atom. For such a system, the effect of a given collision is to shift the energies $E_{\mathrm{b}}$ and $E_{\mathrm{a}}$ of the upper and lower atomic states. This produces a phase shift $\phi$ of the dipole moment

$$
\phi=\int\left(E_{\mathrm{b}}(t)-E_{\mathrm{a}}(t)-\omega_{0}\right) \mathrm{d} t
$$

Summing over collisions gives the following equation describing the damping and the frequency shift of the dipole moment

$\frac{\mathrm{d}}{\mathrm{d} t}\left\langle\mathrm{~b}\left|\sigma_{\mathrm{A}}\right| \mathrm{a}\right\rangle=-(\gamma+i \eta)\left\langle\mathrm{b}\left|\sigma_{\mathrm{A}}\right| \mathrm{a}\right\rangle$ 
( $\sigma_{\mathrm{A}}$ is the atomic density matrix). The two parameters $\gamma$ and $\eta$ are given by

$$
\begin{aligned}
\gamma+i \eta & =\sum_{\text {coll }}(1-\exp (-i \phi)) \\
\gamma & =\sum_{\text {coll }}(1-\cos \phi) \\
\eta & =\sum_{\text {coll }} \sin \phi
\end{aligned}
$$

The summation over collisions is defined by equation (4.5).

We want now to express the dressed atom relaxation parameters $w, \kappa, \xi$ in terms of $\gamma, \eta$ when $\Omega \tau_{\mathrm{c}} \ll 1$, i.e. when the laser atom coupling can be ignored during the collision (see $\S 2.2$ ). The basic idea of the method is that the $S$ matrix of the dressed atom can thus be computed as if the atom was free. Suppose that the dressed atom is before the collision in the state

$|1, n\rangle=\cos \theta|\mathrm{b}, n\rangle+\sin \theta|\mathrm{a}, n+1\rangle$

(see eq. (3.2)). Because of the condition $\omega_{1} \tau_{\mathrm{c}} \ll 1$, the atom laser coupling (strength $\omega_{1}$ ) cannot induce transitions between the two uncoupled states $|\mathrm{b}, n\rangle$ and $|\mathrm{a}, n+1\rangle$ during the collision time $\tau_{\mathrm{c}}$. The effect of the collision is just to dephase these two states so that, after the collision, $|1, n\rangle$ becomes

$$
\begin{aligned}
|\overline{1, n}\rangle= & \cos \theta|\mathrm{b}, n\rangle \exp \left(-i \phi_{\mathrm{b}, n}\right)+ \\
& +\sin \theta|\mathrm{a}, n+1\rangle \exp \left(-i \phi_{\mathrm{a}, n+1}\right) .
\end{aligned}
$$

Since the two shifts are different, $|\overline{1, n}\rangle$ has a non zero projection on the state $|2, n\rangle$ orthogonal to $|1, n\rangle$ (see eq. (3.2))

$$
\begin{aligned}
\langle 2, n \mid \overline{1, n}\rangle=\cos \theta \sin \theta( & \exp \left(-i \phi_{\mathrm{a}, n+1}\right)- \\
- & \left.\exp \left(-i \phi_{\mathrm{b}, n}\right)\right) .
\end{aligned}
$$

This means that the collision induces a population transfer from $|1, n\rangle$ to $|2, n\rangle$ with a probability

$$
|\langle 2, n \mid \overline{1, n}\rangle|^{2}=2 \cos ^{2} \theta \sin ^{2} \theta\left(1-\cos \phi^{\prime}\right)
$$

with

$$
\phi^{\prime}=\phi_{\mathrm{b}, n}-\phi_{\mathrm{a}, n+1}=\int\left(E_{\mathrm{b}}(t)-E_{\mathrm{a}}(t)-\omega_{\mathrm{L}}\right) \mathrm{d} t .
$$

Summing over collisions finally leads to the expression for the redistribution rate $w$

$$
\begin{aligned}
w=\sum_{\text {coll }}|\langle 2, n \mid \overline{1, n}\rangle|^{2}= \\
=2 \cos ^{2} \theta \sin ^{2} \theta \sum_{\text {coll }}\left(1-\cos \phi^{\prime}\right) .
\end{aligned}
$$

This result shows how the collision which is purely elastic in absence of laser irradiation can induce inelastic transitions between the dressed states by dephasing the two uncoupled states. Furthermore, it gives the relation between $w$ and $\gamma$. Because of the relation $\delta \tau_{\mathrm{c}} \ll 1$, one can indeed identify $\omega_{0}$ and $\omega_{\mathrm{L}}$ during the time $\tau_{\mathrm{c}}$ and get from (4.22) and (4.29)

$$
\phi^{\prime}=\phi \text {. }
$$

It follows from equations $(4.24 b),(4.30)$ and $(4.31)$ that

$$
w=2 \gamma \cos ^{2} \theta \sin ^{2} \theta .
$$

From equations (4.32) and (3.3), $w$ can also be expressed in terms of $\gamma, \omega_{1}$ and $\delta$

$$
w=\gamma \omega_{1}^{2} /\left[2\left(\omega_{1}^{2}+\delta^{2}\right)\right] .
$$

One gets the expression of $\kappa$ and $\xi$ by studying the evolution of the dressed atom coherence $|1, n\rangle\langle 2, n|$. The collision transforms it into $|\overline{1, n}\rangle\langle\overline{2, n}|$ where $|\overline{1, n}\rangle$ is given by (5.5) and $|2, n\rangle$ by a quite similar expression

$$
\begin{aligned}
|\overline{2, n}\rangle= & -\sin \theta|\mathrm{b}, n\rangle \exp \left(-i \phi_{\mathrm{b}, n}\right)+ \\
& +\cos \theta|\mathrm{a}, n+1\rangle \exp \left(-i \phi_{\mathrm{a}, n+1}\right)
\end{aligned}
$$

Summation over collisions gives

$\kappa+i \xi=\sum_{\text {coll }}[1-\langle 1, n \mid \overline{1, n}\rangle\langle\overline{2, n} \mid 2, n\rangle]$

(this is equivalent to equation (4.12)). One easily obtains

$$
\begin{aligned}
& 1-\langle 1, n \mid \overline{1, n}\rangle\langle\overline{2, n} \mid 2, n\rangle= \\
& \quad=\cos ^{4} \theta(1-\exp (-i \phi))+\sin ^{4} \theta(1-\exp (i \phi))
\end{aligned}
$$

(we have used (4.31)). It follows that

$$
\begin{aligned}
\kappa+i \xi & =\cos ^{4} \theta(\gamma+i \eta)+\sin ^{4} \theta(\gamma-i \eta) \\
\kappa & =\gamma\left(\cos ^{4} \theta+\sin ^{4} \theta\right)=\gamma\left(\omega_{1}^{2}+2 \delta^{2}\right) /\left[2\left(\omega_{1}^{2}+\delta^{2}\right)\right] \\
\xi & =\eta\left(\cos ^{4} \theta-\sin ^{4} \theta\right)=-\eta \delta / \Omega .
\end{aligned}
$$

By using the expressions (4.33), (4.38) and (4.39) of $w, \kappa$ and $\xi$, one can express in the domain $\Omega \tau_{\mathrm{c}} \ll 1$ the results of section 3 in terms of $\gamma, \omega_{1}, \delta$ and $\Gamma$ (spontaneous emission rate of the upper level). For example, the absorption of laser photons $A$ can be deduced from equations (3.23), (4.33) and (3.3). One gets

$A=\frac{\Gamma}{2}(\Gamma+2 \gamma) \omega_{1}^{2} /\left[(\Gamma+2 \gamma) \omega_{1}^{2}+2 \Gamma \delta^{2}\right]$

which is nothing but the Karplus Schwinger formula [4] in the secular approximation $\left(\Gamma, \gamma \ll \omega_{1}\right.$ or $\Gamma, \gamma \ll \delta$ ). The results concerning the weights and 
widths of the lines of the fluorescence triplet are the following ones :

$$
\begin{aligned}
J\left(\omega_{\mathrm{L}} \pm \Omega\right) & =\frac{\Gamma}{8} \times \\
\times \omega_{1}^{2}\left[\Gamma \omega_{1}^{2}+2 \gamma(\Omega \mp \delta)^{2}\right] /\left[\Omega^{2}\left(\Gamma\left(\Omega^{2}+\delta^{2}\right)+2 \gamma \omega_{1}^{2}\right)\right] & (4.41) \\
J\left(\omega_{\mathrm{L}}\right) & =\frac{\Gamma}{4} \omega_{1}^{2} / \Omega^{2} \\
J_{\mathrm{el}} & =\Gamma^{3} \omega_{1}^{2} \delta^{2} /\left[(\Gamma+2 \gamma) \omega_{1}^{2}+2 \Gamma \delta^{2}\right]^{2} \\
L_{\mathrm{c}} & =\left[\frac{\Gamma}{2}\left(\omega_{1}^{2}+2 \delta^{2}\right)+\gamma \omega_{1}^{2}\right] / \Omega^{2} \\
L_{\mathrm{L}} & =\left[\frac{\Gamma}{4}\left(3 \omega_{1}^{2}+2 \delta^{2}\right)+\frac{\gamma}{2}\left(\omega_{1}^{2}+2 \delta^{2}\right)\right] / \Omega^{2} .
\end{aligned}
$$

We give now a geometrical interpretation of the results obtained for the widths $L_{\mathrm{c}}$ and $L_{\mathrm{L}}$. These widths are actually the damping rates of the components of the fictitious spin respectively parallel and orthogonal to the direction of the effective field $\left(L_{\mathrm{c}}=1 / T_{1}\right.$; $L_{\mathrm{L}}=1 / T_{2}$; see $\S 2.3$ and $\S 3.4$ ). In the domain $\Omega \tau_{\mathrm{c}} \ll 1$, the relaxation is not modified by the laser irradiation and it can be described by optical Bloch equations. The components $S_{Z}, S_{X}$ and $S_{Y}$ of the spin in the $\mathrm{O} X Y Z$ frame have damping rates

$$
\begin{aligned}
& \gamma_{Z}=\Gamma \\
& \gamma_{X}=\gamma_{Y}=\frac{\Gamma}{2}+\gamma
\end{aligned}
$$

( $\Gamma$ and $\gamma$ correspond respectively to the radiative and collisional relaxations). During its fast precession around the effective field, the spin averages the various damping rates corresponding to the various directions which are explored. The components of the spin evolving at frequencies $0, \pm \Omega$, i.e. $S_{\zeta}$ and $S^{ \pm}$(defined with respect to $O \zeta$ ), can be written

$$
\begin{aligned}
S_{\zeta} & =S_{Z} \cos \alpha+S_{X} \sin \alpha \\
S^{ \pm} & =\left(S_{X} \cos \alpha-S_{Z} \sin \alpha\right) \pm i S_{Y} .
\end{aligned}
$$

Their damping rates $L_{\mathrm{c}}$ and $L_{\mathrm{L}}$ have therefore the following values

$$
\begin{aligned}
L_{\mathrm{c}} & =\gamma_{Z} \cos ^{2} \alpha+\gamma_{X} \sin ^{2} \alpha \\
& =\Gamma \cos ^{2} \alpha+\left(\frac{\Gamma}{2}+\gamma\right) \sin ^{2} \alpha \\
L_{\mathrm{L}} & =\frac{1}{2}\left(\gamma_{Z} \sin ^{2} \alpha+\gamma_{X} \cos ^{2} \alpha+\gamma_{Y}\right) \\
& =\frac{\Gamma}{2} \sin ^{2} \alpha+\frac{1}{2}\left(\frac{\Gamma}{2}+\gamma\right)\left(1+\cos ^{2} \alpha\right)
\end{aligned}
$$

which are the same results as in (4.44) and (4.45) (since $\sin ^{2} \alpha=\omega_{1}^{2} / \Omega^{2}$ and $\cos ^{2} \alpha=\delta^{2} / \Omega^{2}$ ). This discussion actually gives a geometrical computation of $w$ (which is half the collisional contribution to $L_{\mathrm{c}}$; see equation 3.21) and $\kappa$ (equal to the collisional contribution to $L_{\mathrm{L}}$; see equation (3.17)). The results are the same as in (4.32) and (4.38). This clearly shows that $w$ and $\kappa$ are related to $\gamma$ in the domain $\Omega \tau_{\mathrm{c}} \ll 1$ by simple « geometrical factors ».

Remark on a problem of terminology. - Condition $\Omega \tau_{\mathrm{c}} \ll 1$ defines a situation which is often called the « impact region " $[15,17,20,22,23,24]$. On the other hand, the word impact also appears in the « impact approximation " which, in pressure broadening theory $[6,8]$, corresponds to a situation where strong collisions are well separated in time $\left(\gamma \tau_{\mathrm{c}} \ll 1\right)$. With such an approximation, the results obtained for absorption or emission spectra are restricted to some frequency interval $\Delta \omega \tau_{\mathrm{c}} \ll 1(\Delta \omega$ distance from the centre of the line) which can thus be called the « impact domain ". These three expressions using the word impact correspond to different physical ideas. For example, in this paper, we use, even outside the "impact region" $\left(\Omega \tau_{\mathrm{c}}>1\right)$, the impact approximation $\left(\gamma \tau_{\mathrm{c}} \ll 1\right)$, which leads to the impact domain associated with the three intervals of figure 4.

There is however a risk of confusion and some authors $[28,32]$ suggest that one should refer to the situation $\Omega \tau_{\mathrm{c}} \ll 1$ as "medium coupling " and $\Omega \tau_{\mathrm{c}}>1$ as "strong coupling ". Actually such terminology is also questionable since condition $\Omega \tau_{\mathrm{c}}>1$ corresponds, in most experimental cases, to $\delta \tau_{\mathrm{c}}>1$ whereas $\omega_{1} \tau_{\mathrm{c}}<1$. It seems strange to call strong coupling a situation where the Rabi frequency remains small.

In our opinion, a terminology avoiding the previous disadvantages should refer to the main feature of the domain $\Omega \tau_{\mathrm{c}} \ll 1$, i.e. the independence of the collisional relaxation from the laser irradiation.

4.4 Recapitulation. - We now summarize the results of this section 4 . The relaxation equations (3.9) and (3.10) neglect non Markovian as well as non secular effects. The redistribution spectrum deduced from these equations is therefore limited to three intervals

$\left|\omega-\left(\omega_{\mathrm{L}}+\alpha \Omega\right)\right| \ll$ smaller of $\left(\tau_{\mathrm{c}}\right)^{-1}$ and $\Omega$.

But the main features of this spectrum (i.e. the positions, widths and weights of the lines) as well as the net absorption of laser photons are correctly given by the relaxation equations when

$$
\gamma \ll \operatorname{smaller} \text { of }\left(\tau_{\mathrm{c}}\right)^{-1} \text { and } \Omega .
$$

In the domain $\Omega \tau_{\mathrm{c}} \ll 1$, the parameters $w, \kappa, \xi$ and therefore all the results of section 3 can be expressed in terms of the bare atom parameters $\gamma$ and $\eta$. These results are identical to the results derived from optical Bloch equations [19]. In this domain, the secular 
approximation is more questionable than the Markov one (in equations (4.52) and (4.53), $\Omega$ is smaller than $\left.\tau_{\mathrm{c}}^{-1}\right)$. If necessary, it is possible to take into account the non secular terms. This is equivalent to deriving the spectrum from the optical Bloch equations without any secular approximation.

On the other hand, if $\Omega \tau_{\mathrm{c}}>1$, the Markov approximation is more critical than the secular one $\left(\tau_{\mathrm{c}}^{-1}\right.$ is smaller than $\Omega$ ). In this case, it is not worthwhile to try to study the effect of the non secular terms.

5. Relation with the relaxation of a spin in a fluctuating field. - We come back now to the spin representation of section 2 and try to formulate the $T_{1}$ and $T_{2}$ type relaxation parameters $w$ and $\kappa$ in terms of some correlation functions of the fluctuating field $h(t)$ describing the effect of collisions [7, 10].

We will consider first $(\$ 5.1)$ a simple situation where the effect of the collisional field $h(t)$ can be studied in the so called « motional narrowing limit » $\left(h \tau_{\mathrm{c}} \ll 1\right)$ corresponding to weak collisions (the phase shift $h \tau_{\mathrm{c}}$ produced by every elementary collision is small). Such a model is not realistic since strong collisions $\left(h \tau_{\mathrm{c}}>1\right)$ play actually an important role, but it leads to simple and suggestive expressions. We will then extend $(\S 5.2)$ the structure of some of these expressions to the strong collisions case.

5.1 THE WEAK COLLISIONS LIMIT. - The expressions giving, in the motional narrowing limit, the relaxation parameters in terms of correlation functions of the fluctuating perturbation are well known [39, 43].

As discussed in section 2 , the $T_{1}$ relaxation is produced by $h_{\perp}(t)=h(t) \sin \alpha$ (see Fig. 2). On the other hand, the adiabatic contribution $\kappa^{\prime}$ to the $T_{2}$ relaxation ( $T_{2}^{\prime}$ type relaxation; see $\left.\S 4.1\right)$ is produced by $h_{\|}(t)=h(t) \cos \alpha$ (see Fig. 2). The relaxation parameters can therefore be expressed in terms of the correlation functions $C(\tau)$ of the field $h(t)$

$$
C(\tau)=\sum_{\text {coll }} \int_{-\infty}^{+\infty} \mathrm{d} t h(t) h(t+\tau)
$$

or more precisely in terms of the Fourier Laplace transform of $C(\tau)$

$$
\begin{aligned}
J(\omega) & =\operatorname{Re}\left[\int_{0}^{\infty} \mathrm{d} \tau \mathrm{e}^{-i \omega \tau} C(\tau)\right] \\
& =\frac{1}{2} \int_{-\infty}^{+\infty} \mathrm{d} \tau \mathrm{e}^{-i \omega \tau} C(\tau)
\end{aligned}
$$

The results obtained in this way

$$
\begin{aligned}
& 1 / T_{1}=2 w=\sin ^{2} \alpha J(\Omega) \\
& 1 / T_{2}^{\prime}=\kappa^{\prime}=\cos ^{2} \alpha \mathrm{J}(0)
\end{aligned}
$$

have the usual physical interpretation : the $T_{1}$ relaxation parameter $w$, which describes transitions between two spin states separated by $\Omega$, is equal to the Fourier transform at the frequency $\Omega$ of the correlation function $\sin ^{2} \alpha C(\tau)$ of $h_{\perp}(t)$, whereas the $T_{2}^{\prime}$ adiabatic parameter $\kappa^{\prime}$ is the Fourier transform at the frequency 0 of the correlation function $\cos ^{2} \alpha C(\tau)$ of $h_{\|}(t)$. The same theory gives, when applied to the bare atom, a pure adiabatic relaxation (see $\S 2.1$ ) described by

$$
\gamma=J(0) \text {. }
$$

Using these results, we can now explicit the discussion of $\S 2.2$ about the importance of the parameter $\Omega \tau_{\mathrm{c}}$. The width of $C(\tau)$ being of the order of the collision time $\tau_{\mathrm{c}}$, it follows that the width of its Fourier transform $J(\omega)$ is of the order of $\tau_{\mathrm{c}}^{-1}$. Suppose first $\Omega \tau_{\mathrm{c}} \ll 1$ (i.e. $\Omega \ll \tau_{\mathrm{c}}^{-1}$ ). In this case, $J(\Omega) \simeq J(0)$ and it clearly appears on (5.3), (5.4) and (5.5) that $w$ and $\kappa\left(\kappa=\kappa^{\prime}+w\right.$; see eq. (4.15)) are related to $\gamma$ by simple geometrical factors (same results as in $\S 4.3)$. On the other hand, if $\Omega>\tau_{\mathrm{c}}^{-1}, J(\Omega)$ and $J(0)$ have quite different values and the parameters $w$ and $\kappa$ are no longer simply related to $\gamma$. In other words, $w$ and $\kappa^{\prime}$ contain different informations about the collision dynamics since the difference between the frequencies 0 and $\Omega$ is larger than $\tau_{\mathrm{c}}^{-1}$.

It will be also interesting to discuss the situation $\delta \tau_{\mathrm{c}}>1, \omega_{1} \tau_{\mathrm{c}} \ll 1$ which is the easiest way for obtaining experimentally $\Omega \tau_{\mathrm{c}}>1$ [17]. At first sight, one could think that the laser atom coupling can be ignored during the collision (since $\omega_{1} \tau_{\mathrm{c}} \ll 1$ ). But expression (5.3) reduces here to

$$
1 / T_{1}=2 w=\left(\omega_{1}^{2} / \delta^{2}\right) J(\delta)
$$

which cannot be related to $\gamma$ since $J(\delta) \neq J(0)$. The important point is that $w$ describes transitions between states separated by $\delta$ and that, from energy conservation, $w$ depends on Fourier components of the collisional perturbation at a frequency $\delta$ larger than $\tau_{c}^{-1}$. This is precisely why the experimental study of far wing absorption is interesting : the deviation from the lorentzian shape gives access to the variation of $J(\delta)$ with $\delta$.

5.2 EXTENSION TO STRONG COLLISIONS. - In the case of strong collisions $\left(h \tau_{\mathrm{c}}>1\right)$, it might seem impossible to write the relaxation parameters as Fourier transforms of some simple correlation functions. In this section, we point out that such a mathematical structure which generalizes the results of the previous section, still exists for $\gamma$ in the general case and for $w$ and $\kappa$ in the situations having presently an experimental importance (" impact region »: $\Omega \tau_{\mathrm{c}} \ll 1$ and far wing weak excitation $: \delta \tau_{\mathrm{c}}>1, \omega_{1} \ll \delta$ ).

5.2.1 Expression of $\gamma$. - In the appendix, we show that the expression (4.24b) of the damping rate $\gamma$ of the bare dipole moment can be transformed into

$$
\gamma=\tilde{J}(0)
$$


where $\widetilde{J}(0)$ is the value, at frequency 0 , of the Fourier transform of the correlation function

$$
\tilde{C}(\tau)=\sum_{\text {coll }} \int_{-\infty}^{+\infty} h(t) \mathrm{e}^{-i \psi(t)} h(t+\tau) \mathrm{e}^{i \psi(t+\tau)} \mathrm{d} t
$$

with

$$
\psi(t)=\int_{-\infty}^{t} h\left(t^{\prime}\right) \mathrm{d} t^{\prime}
$$

Expression (5.9) generalizes for strong collisions the expression (5.1) of $C(\tau)$ by including the phase shift $\psi(t+\tau)-\psi(t)$ produced by the collision between $t$ and $t+\tau$ (for weak collisions this phase shift, of the order of $h \tau_{\mathrm{c}}$, can be ignored and $\tilde{C}(\tau)$ reduces to $C(\tau))$.

5.2.2 Expression of $w$ and $\kappa^{\prime}$ when $\Omega \tau_{\mathrm{c}} \ll 1$. It has been shown above (see $\S 4.3$ ) that $w$ and $\kappa$ are related to $\gamma$ by simple geometrical factors when $\Omega \tau_{\mathrm{c}} \ll 1$. It follows that

$$
\begin{aligned}
& 1 / T_{1}=2 w=\gamma \sin ^{2} \alpha=\sin ^{2} \alpha \tilde{J}(0) \\
& 1 / T_{2}^{\prime}=\kappa^{\prime}=\gamma \cos ^{2} \alpha=\cos ^{2} \alpha \widetilde{J}(0) .
\end{aligned}
$$

This generalizes the results (5.3) and (5.4) of the motional narrowing limit provided that $J$ is replaced by $\widetilde{J}\left(\right.$ here $\widetilde{J}(0)=\widetilde{J}(\Omega)$ since $\left.\Omega \tau_{\mathrm{c}} \ll 1\right)$.

5.2.3 Expression of $w$ and $\kappa^{\prime}$ when $h_{\perp} \tau_{c} \ll 1$. Another situation leading to simple expressions for $w$ and $\kappa^{\prime}$ occurs when the effect of $h_{\perp}$ can be treated perturbatively during the collision time, i.e. when $h_{\perp} \tau_{\mathrm{c}} \ll 1$. Since $h_{\perp}=h \sin \alpha$, condition $h_{\perp} \tau_{\mathrm{c}} \ll 1$ can be verified for strong collisions $\left(h \tau_{\mathrm{c}}>1\right)$ provided that $\sin \alpha \ll 1$, i.e. $\omega_{1} \ll \delta$ (weak excitation limit). The perturbative treatment of $h_{\perp}$ leads to the following result for the redistribution rate $w$ (see Appendix)

$$
1 / T_{1}=2 w=\sin ^{2} \alpha \widetilde{J}(\delta)=\left(\omega_{1}^{2} / \delta^{2}\right) \widetilde{J}(\delta)
$$

where $\widetilde{J}(\delta)$ is the Fourier transform at frequency $\delta$ of the correlation function $\tilde{C}(\tau)$ given in (5.8). The adiabatic relaxation parameter $\kappa^{\prime}$ associated with $h_{\|}$ is equal to $\gamma\left(\right.$ since $h_{\|}=h \cos \alpha \simeq h$ if $\omega_{1} \ll \delta$ )

$$
1 / T_{2}^{\prime}=\kappa^{\prime}=\tilde{J}(0)
$$

These results are valid for $\delta \tau_{\mathrm{c}} \ll 1$ as well as for $\delta \tau_{\mathrm{c}}>1$ (condition $h_{\perp} \tau_{\mathrm{c}} \ll 1$ can occur in the two situations). When $\delta \tau_{\mathrm{c}} \ll 1$ (which is equivalent to $\Omega \tau_{\mathrm{c}} \ll 1$ since $\left.\omega_{1} \ll \delta\right)$ they coincide with the expressions (5.10) and (5.11) (taken in the limit $\sin \alpha \ll 1$ ). More interesting is the other case since it corresponds to the situation experimentally accessible of far wing weak excitation $\left(\delta \tau_{c}>1\right.$ and $\left.\omega_{1} \ll \delta\right)$. From equation (5.12) and from the results of section 3 , one can get the weight of the fluorescent line and the absorption of laser photons in terms of $\widetilde{J(\delta)}$ :

$$
\begin{aligned}
& \mathfrak{J}\left(\omega_{0}\right)=w=\tilde{J}(\delta) \omega_{1}^{2} / 2 \delta^{2} \\
& A=\left(\frac{\Gamma}{2}+\tilde{J}(\delta)\right) \omega_{1}^{2} / 2 \delta^{2} .
\end{aligned}
$$

The quantity $\tilde{J}(\delta)$ thus appears as identical to the phenomenological parameter $\gamma(\delta)$ introduced by Carlsten, Szoke and Raymer in their experimental paper [17]. The approach developed in this section thus allows us to interpret this phenomenological parameter $\gamma(\delta)$ as the Fourier transform at frequency $\delta$ of the correlation function $\tilde{C}(\tau)$ defined by $(5.8)$.

Remark. - It might be tempting to consider $\gamma(\delta)=\tilde{\boldsymbol{J}}(\delta)$ as an "effective relaxation parameter" replacing $\gamma=\tilde{J}(0)$ in optical Bloch equations. It clearly appears from the previous discussion that this method would not be correct. As a matter of fact, it would lead to a good result for $w$ and therefore for the weight of the fluorescent line and the absorption but to a wrong result for $\kappa^{\prime}$ and therefore for the line widths of the two sidebands since $\kappa^{\prime}$ is related to $\widetilde{J}(0)$. Actually, optical Bloch equations may be generalized when $\delta \tau_{\mathrm{c}}>1$ by introducing a $\delta$ dependence in the relaxation matrix $\mathfrak{F}_{\text {coll }}$ of equation (1.1) (and also an $\omega_{1}$ dependence for $\omega_{1} \tau_{c}>1$ ) [40]. But such generalized optical Bloch equations must involve the two quantities $\widetilde{J}(0)$ and $\tilde{J}(\delta)$ which are needed to describe the effect of collisions.

6. Conclusion. - We have presented in this paper a dressed atom approach to collisional effects in resonance fluorescence which provides an interpretation of collisional redistribution in terms of collision induced transition rates between the dressed states. All the characteristics of absorption and emission spectra have been related to $T_{1}$ and $T_{2}$ type relaxation parameters of the dressed atom. Such an approach generalizes the usual perturbative picture (Fig. 1) to high intensity and resonant situations.

Furthermore, when compared with « optical Bloch's equations ", the dressed atom approach not only provides a simpler physical insight but appears to have a larger domain of validity. We have shown that it gives a correct Markovian description of absorption and of spectral redistribution even when optical Bloch's equations are no longer valid $\left(\Omega \tau_{\mathrm{c}} \gtrsim 1\right)$.

The structure of the expressions giving the relaxation parameters of the dressed atom have been also investigated. They have been first related to the collision $S$ matrix. By using a spin representation of the problem, we have also shown that they can be expressed, in most cases, in terms of some correlation functions of the collisional perturbation. Furthermore, such a spin representation has provided interesting 
geometrical insights concerning the separation of the two domains where the collisional relaxation depends $\left(\Omega \tau_{\mathrm{c}}>1\right)$ or does not depend $\left(\Omega \tau_{\mathrm{c}} \ll 1\right)$ on the laser irradiation.

This paper has been limited to the simplest case of two level atoms. But the dressed atom approach presented here would be even more suited to more complicated systems. For example, it has been used for interpreting some resonances between unpopulated levels in non linear optics [41].

Appendix. - (i) The expression (4.24b) of $\gamma$ can be transformed into

$$
\gamma=\frac{1}{2} \sum_{\text {coll }}\left(\mathrm{e}^{-i \phi}-1\right)\left(\mathrm{e}^{i \phi}-1\right) .
$$

Then, it can be noted that

$$
\begin{aligned}
\mathrm{e}^{-i \phi}-1 & =\left[\mathrm{e}^{-i \psi(t)}\right]_{-\infty}^{+\infty} \\
& =\int_{-\infty}^{+\infty}-i h(t) \mathrm{e}^{-i \psi(t)} \mathrm{d} t \\
\mathrm{e}^{i \phi}-1 & =\int_{-\infty}^{+\infty} i h\left(t^{\prime}\right) \mathrm{e}^{i \psi\left(t^{\prime}\right)} \mathrm{d} t^{\prime}
\end{aligned}
$$

(where $\psi(t)$ is defined by (5.9); one has used $h(t)=\mathrm{d} \psi / \mathrm{d} t)$. Inserting (A.2) and (A.3) into (A .1) and introducing the variable $\tau=t^{\prime}-t$ leads to

with

$$
\gamma=\frac{1}{2} \int_{-\infty}^{+\infty} \mathrm{d} \tau \tilde{C}(\tau)
$$

$$
\widetilde{C}(\tau)=\sum_{\text {coll }} \int_{-\infty}^{+\infty} h(t) h(t+\tau) \mathrm{e}^{-i \psi(t)} \mathrm{e}^{i \psi(t+\tau)} \mathrm{d} t .
$$

Equation (A.4) is equivalent to equation (5.7)

with

$$
\begin{gathered}
\gamma=\widetilde{J}(0) \\
\tilde{J}(\omega)=\frac{1}{2} \int_{-\infty}^{+\infty} \mathrm{d} \tau \mathrm{e}^{-i \omega \tau} \tilde{C}(\tau) .
\end{gathered}
$$

(ii) The equations describing transitions between the dressed states during a given collision are well known (see for example reference [15] eq. (2.7)).

Their perturbative resolution when $h_{\perp} \tau_{\mathrm{c}} \ll 1$ gives

$$
\left|S_{21}\right|^{2}=\left|\frac{\sin \alpha}{2} \int_{-\infty}^{+\infty} h(t) \exp [i(\Omega t-\psi(t) \cos \alpha)] \mathrm{d} t\right|^{2} .
$$

We will suppose also $\omega_{1} \ll \delta(\sin \alpha \ll 1, \cos \alpha= \pm 1)$. Since $\Omega=\delta / \cos \alpha$, (A.8) can be written in the two cases $\cos \alpha= \pm 1(\delta>0$ or $\delta<0)$ as

$$
\begin{aligned}
\left|S_{21}\right|^{2} & =\frac{\sin ^{2} \alpha}{4}\left|\int_{-\infty}^{+\infty} h(t) \exp [i(\delta t-\psi(t))] \mathrm{d} t\right|^{2} \\
& =\frac{\sin ^{2} \alpha}{4} \int_{-\infty}^{+\infty} h(t) \mathrm{e}^{i(\delta t-\psi(t))} \mathrm{d} t \int_{-\infty}^{+\infty} h\left(t^{\prime}\right) \mathrm{e}^{-i\left(\delta t^{\prime}-\psi\left(t^{\prime}\right)\right)} \mathrm{d} t^{\prime}
\end{aligned}
$$

Summing over collisions and introducing $\tau=t^{\prime}-t$ leads to

$$
\begin{aligned}
w=\sum_{\text {coll }}\left|S_{21}\right|^{2} & =\frac{\sin ^{2} \alpha}{4} \int_{-\infty}^{+\infty} \mathrm{d} \tau \mathrm{e}^{-i \delta \tau} \widetilde{C}(\tau) \\
& =\frac{\sin ^{2} \alpha}{2} \widetilde{J}(\delta)
\end{aligned}
$$

(which is equivalent to (5.12)). 


\section{References}

[1] Lorentz, H. A., Proc. Amst. Acad. Sci. 8 (1906) 59.

[2] Weisskopf, V., Z. Phys. 75 (1932) 287.

[3] Lindholm, E., Arkiv. Mat. Astron. Fysik 28B (1941) $\mathrm{n}^{\mathrm{o}} 3$.

[4] Karplus, R. and Schwinger, J., Phys. Rev. 73 (1948) 1020.

[5] Anderson, P. W., Phys. Rev. 76 (1949) 647.

[6] Baranger, M., Phys. Rev. 111 (1958) 481.

[7] KuBo, R., in " Fluctuation, Relaxation and Resonance in Magnetic Systems ", D. ter Haar ed., (Oliver and Boyd, Edinburgh) 1961, p. 23.

[8] Fiutak, J. and Van Kranendonk, J., Can. J. Phys. 40 (1962) 1085.

[9] Fano, U., Phys. Rev. 131 (1963) 259.

[10] Huber, D. L., Phys. Rev. 170 (1968) 418.

[11] Huber, D. L., Phys. Rev. 178 (1969) 93.

[12] Mollow, B. R., Phys. Rev. A2 (1970) 76.

[13] Omont, A., Smith, E. W. and CoOPER, J., Astrophys. J. 175 (1972) 185.

[14] Pestov, E. G. and Rautian, S. G., Sov. Phys. JetP 37 (1973) 1025.

[15] Lisitsa, V. S. and Yakovlenko, S. I., Sov. Phys. JETP 41 (1975) 233.

[16] Andreev, S. P. and Lisitsa, V. S., Sov. Phys. JETP 45 (1977) 38.

[17] Carlsten, J. L., Szoke, A. and Raymer, M. G., Phis. Rev. A15 (1977) 1029.

[18] Courtens, E. and Szoke, A., Phys. Rev. A15 (1977) 1588.

[19] Mollow, B. R., Phys. Rev. A15 (1977) 1023.

[20] Nienhuis, G. and Schuller, F., Physica 92C (1977) 397.

[21] Light, J. C. and Szoke, A., Phys. Rev. A18 (1978) 1363.

[22] Voslamber, D. and Yelnik, J. B., Phys. Rev. Lett. 41 (1978) 1233.

[23] Yeh, S. and Berman, P. R., Phys. Rev. A19 (1979) 1106.
[24] CoOper, J., Astrophys. J. 228 (1979) 339.

[25] Mukamel, S., J. Chem. Phys. 71 (1979) 2884

[26] Nienhuis, G. and Schuller, F., J. Phys. B12 (1979) 3473.

[27] FiutaK, J. and Van Kranendonk, J., J. Phys. B13 (1980) 2869.

[28] Rabin, Y. and Ben-Reuven, A., J. Phys. B13 (1980) 2011.

[29] Bonch-Bruevich, A. M., Vartanyan, T. A. and Khromov, V. V., Sov. Phys. JETP 51 (1980) 271

[30] Schuller, F. and Nienhuis, G., Physica 103C (1981) 369.

[31] ZAIDI, H. R., Can. J. Phys. 59 (1981) 750

[32] Burnett, K., Cooper, J., Kleiber, P. D. and BenReuven, A., Phys. Rev. A25 (1982) 1345.

[33] Rautian, S. G. and Sobelman, 1. 1., Sov. Phys. JetP 14 (1962) 328.

[34] Mollow, B. R., Phys. Rev. 188 (1969) 1969.

[35] Cohen-Tannoudj, C. and Reynaud, S., in "Multiphoton Processes " J. H. Eberly and P. Lambropoulos ed., (Wiley, New York) 1978, p. 103.

[36] Cohen-Tannoudi, C. and Reynaud, S., J. Phys. B10 (1977) 345.

[37] Cohen-Tannoudj,, C., in "Laser Spectroscopy " S. Haroche, J. C. Pebay Peyroula, T. W. Hansch and S. E. Harris ed., (Springer, Berlin) 1975, p. 324.

[38] Lax, M., Phys. Rev. 172 (1968) 350.

[39] Abragam, A., The principles of nuclear magnetism (Oxford University Press, London) 1961.

[40] Bloch, F., Phys. Rev. 105 (1957) 1206.

[41] Grynberg, G., J. Phys. B14 (1981) 2089.

[42] Reynaud, S. and Cohen-Tannoudj, C., in Laser Spectroscopy V, A.R.W. Mc Kellar, T. Oka, B. P. Stoïcheff ed., (Springer Verlag, Berlin) 1981 p. 166.

[43] Slichter, C. P., Principles of Magnetic Resonance, (Harper and Row, New York) 1963. 